\title{
Gamma-ray Imaging Performance of Nuclear Emulsion Telescope in GRAINE-2018 Balloon Experiment
}

\author{
Hiroki Rokujo*, Masahiro Komiyama, Yuya Nakamura \\ Nagoya University \\ E-mail: rokujodflab.phys.nagoya-u.ac.jp
}

\section{Saya Yamamoto}

Okayama University of Science

\section{for GRAINE collaboration}

Aichi University of Education, ISAS/JAXA, Kobe University, Nagoya University, and Okayama

University of Science

Since 2008, the Large Area Telescope onboard the Fermi Gamma-ray Space Tele- scope (FermiLAT) has surveyed the sub-GeV/GeV gamma-ray sky and achieved high statistics measurements. However, observation at low galactic latitudes remains difficult owing to the lack of the angular resolution, and new issues following the operation of Fermi-LAT have arisen. We devised a precise gamma-ray observation project, Gamma-Ray Astro-Imager with Nuclear Emulsion (GRAINE), using balloon-borne emulsion gamma-ray telescopes to realize high angular resolution, polarization-sensitive, and large-aperture observations in the $0.01-100 \mathrm{GeV}$ energy region. On April 26, 2018, the 3rd balloon experiment of the GRAINE project was conducted in Alice Springs, Australia, to detect celestial gamma-ray sources and to demonstrate the overall performance of the middle-sized emulsion telescope (aperture area of $0.4 \mathrm{~m}^{2}$ ). The balloon floated at the altitude of 36-38 km for $15 \mathrm{~h}$, and the telescope observed the target object, Vela pulsar, for 6 h. Following the recovery and the photofinishing, the data acquisition by the emulsion scanning system were completed, and then the flight data analysis has been performed using reconstructed gamma-ray events. In this presentation, the in-flight performance focusing on the converter part of the emulsion telescope employed in the GRAINE 2018 balloon experiment is reported.

36th International Cosmic Ray Conference -ICRC2019-

July 24th - August 1st, 2019

Madison, WI, U.S.A.

\footnotetext{
* Speaker.
} 


\section{Introduction}

Since 2008, the Large Area Telescope onboard the Fermi Gamma-ray Space Telescope (FermiLAT) [四] has surveyed the sub-GeV/GeV gamma-ray sky and achieved high statistics measurements. However, observation at low galactic latitudes remains difficult owing to the lack of the angular resolution, and new issues following the operation of Fermi-LAT have arisen; many gammaray sources remain unassociated on the galactic plane due to source confusions [ [व]; unexpected gamma-ray excess in the galactic center region has been reported [3]. Recently, the future projects focusing on the improvement of the angular resolution are proposed [ [⿴囗十, [1, 目].

Gamma-Ray Astro-Imager with Nuclear Emulsion (GRAINE) is a precise observation project of cosmic gamma rays in the $0.01-100 \mathrm{GeV}$ energy region with a balloon-borne large-aperture-area telescope based on nuclear emulsion technology [ [] . Nuclear emulsion is a tracking detector that can observe three-dimensional (3D) trajectories of charged particles with sub-micron resolution and thin material ( $\sim 0.1 \%$ of the radiation length unit). It can precisely determine an incident angle of gamma ray by measuring electron and positron tracks via the pair creation $\left(\gamma+(\gamma) \rightarrow \mathrm{e}^{+}+\mathrm{e}^{-}\right)$ at the conversion point. The angular resolutions of the emulsion gamma-ray telescope for the gamma-ray energies of $0.1 \mathrm{GeV}$ and $1 \mathrm{GeV}$ are $1^{\circ}$ and $0.1^{\circ}$, respectively. These performances are about one order of magnitude better than those of Fermi-LAT. Moreover, nuclear emulsion realizes a significant measurement of the azimuthal angle of the plane where electron and positron tracks lie, and has sensitivity for polarized gamma rays [ [8, []. The features of nuclear emulsion are also dead space free and lightweight. It is the only detector which realizes to expand the aperture area while maintaining the resolution. GRAINE repeats the observation by balloon-borne experiment with updating the detector scale, and aims at developing into science observation using full scale telescope (aperture area of $10 \mathrm{~m}^{2}$ ).

Until now, we have developed various detector components using accelerator beam tests (gamma rays at SPring-8 and UVSOR, muons at Muon Pit of the neutrino beam line in J-PARC, electrons

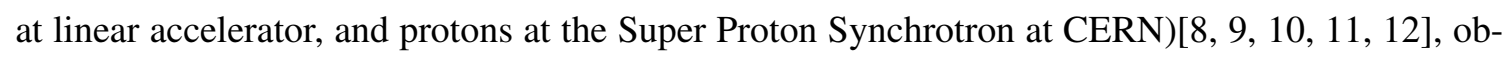
servations at ground and mountain levels (Norikura) [8, [13], and three balloon-borne experiments. We verified the feasibility of the observation by a small-scale emulsion telescope (aperture area of $0.013 \mathrm{~m}^{2}$ ) in the first balloon experiment, GRAINE 2011 [प4, [5]], and then we demonstrated the flight performance using a middle-scale telescope $\left(0.38 \mathrm{~m}^{2}\right)$ in the second balloon experiment, GRAINE 2015 [16, [1], [18].

April 26, 2018, the 3rd balloon experiment of the GRAINE project, GRAINE 2018 [प⿴囗, 20], was conducted in Alice Springs, Australia, to detect Vela pulsar, which is the brightest gamma-ray source in the sub-GeV-GeV region, and to demonstrate the overall performance of the middlesized emulsion telescope. In this paper, the in-flight performance focusing on the converter part of the emulsion telescope is reported. The experimental apparatus and the balloon flight are briefly introduced in section 2. The analysis using scanned emulsion track data is described in section 3. The gamma-ray imaging performance is evaluated and discussed in section 4 . 


\section{GRAINE 2018 Balloon Experiment}

\subsection{Detector}

The GRAINE 2018 payload mainly consists of a pressure vessel gondola, star cameras, and the emulsion gamma-ray telescope. The left photograph in figure $\mathbb{W}$ show the flight-ready payload suspended by a crane truck. The cylindrical-shape container is the balloon-style pressure vessel, which enables to maintain robust and vacuum-packed emulsion chambers even at balloon altitudes [ [2]]; the greater part of the vessel is made of membrane materials consisting of ultra-thin polyurethane-coated polyester fabric. The star camera system monitors the attitude of the emulsion telescope during the observation. It consists of three far infrared cameras with hoods avoiding stray light, which were placed on the gondola toward deference directions, captured and stored images of stars continuously.

The center photograph in figure $\mathbb{W}$ shows the emulsion telescope inside of the pressure vessel gondola. The components of the GRAINE 2018 emulsion telescope are the converter, the time stamper, and the alignment unit. The right-hand pane in figure $\mathbb{W}$ shows a cross sectional view of the emulsion telescope. The converter consists of 100-stacked emulsion films, which were developed and manufactured at Nagoya University. A film has $75-\mu \mathrm{m}$-thick emulsion layers on both sides of a 180- $\mu$ m-thick polystyrene film. The thickness and radiation length are $33 \mathrm{~mm}$ and $0.52 X_{0}$, respectively. $33 \%$ of vertical incident gamma rays convert electron-positron tracks and the pair tracks are recorded in emulsion layers. The size of a film was $37.8 \mathrm{~cm} \times 25 \mathrm{~cm}$ and 4 units of converter were placed at the detector mounting area of the gondola. The aperture area corresponds to $0.38 \mathrm{~m}^{2}$. Alignment films, which were vacuum-packed with a flat aluminum honeycomb panel, were placed at the most up-steam part of each unit. The angle calibration is conducted by connecting high-momentum tracks between alignment films and converter films. Time stamper was set at the bottom of the converter and consists of the multi-stage shifter [22]. It has 4 stages and 2-3 emulsion films were placed on each stages. Films that has $50 \mu \mathrm{m}$-thick emulsion layers were employed for the time stamper. Three stages except the most up-stream part were slid and controlled by stepping motors.
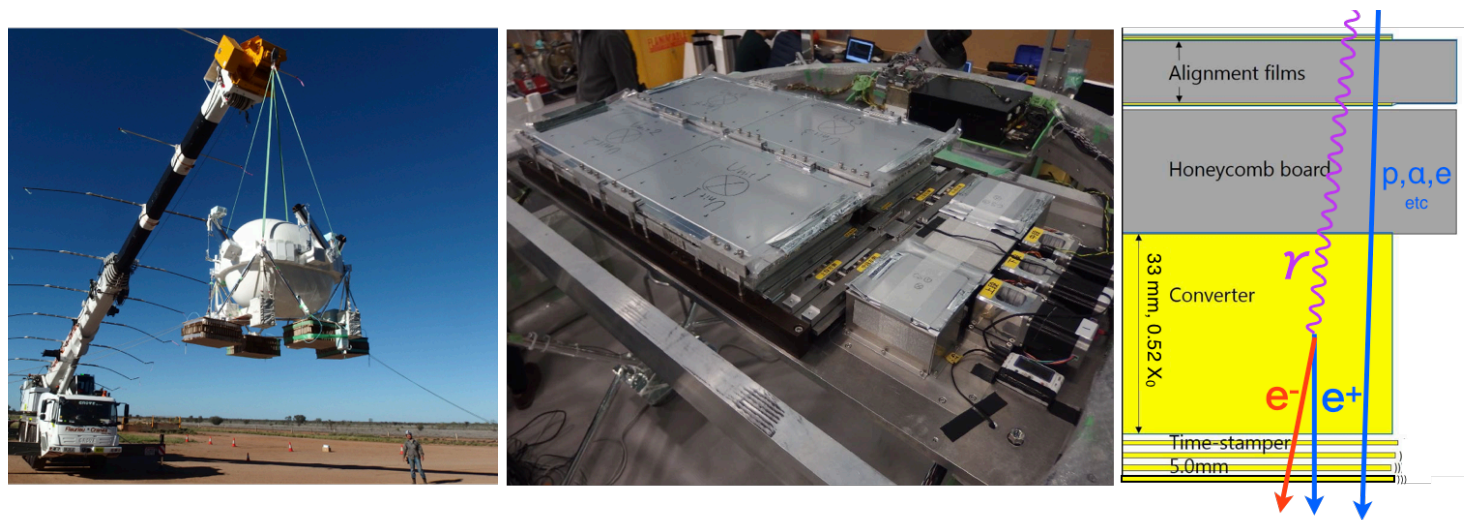

Figure 1: The left and center photographs show the flight-ready payload suspended by a crane truck, and the emulsion telescope inside of the pressure vessel gondola, respectively, in the GRAINE 2018 experiment. The right figure shows a cross sectional view of the emulsion telescope. 


\subsection{Balloon Flight}

Collaborating GRAINE members arrived in the Alice Springs Balloon Launching Station, Australia on March 1, 2018. The assembly of the payload, operation test, and final compatibility check were completed by March 30. On April 26, the wind speed and direction predictions satisfied the required conditions, and the balloon launch was performed at 6:33 Australian Central Standard Time (ACST). The balloon reached an altitude of $38 \mathrm{~km}$ in $2 \mathrm{~h}$ and started level flight heading eastward. The emulsion telescope and the three star cameras were operating stably and the observation continued until 22:19 ACST. The Vela pulsar crossed a field of view of the telescope for approximately $7 \mathrm{~h}$ and the observation was successfully performed as planned. The balloon released the gondola equipped with a parachute at 23:17 ACST, which landed on the ground 250 $\mathrm{km}$ southwest of Longreach at 23:54 ACST.

The recovery of the payload was completed using a helicopter on the next day, and emulsion films were transported to Sydney immediately. The development of all films was processed at the University of Sydney. All processes were finished by May 13.

\section{Flight Data Analysis}

\subsection{Data acquisition}

After cleaning surface silver and swelling emulsion layers, track data recored in flight emulsion films were readout by the automatic scanning system, Hyper Track Selector (HTS) [23], which was developed at Nagoya University. Figure $\square$ shows a progress in the data acquisition of converter films. The HTS machine time of approximately 30 hours was obtained every week; data scanning for converter films have run for 20 weeks and finished without accident.

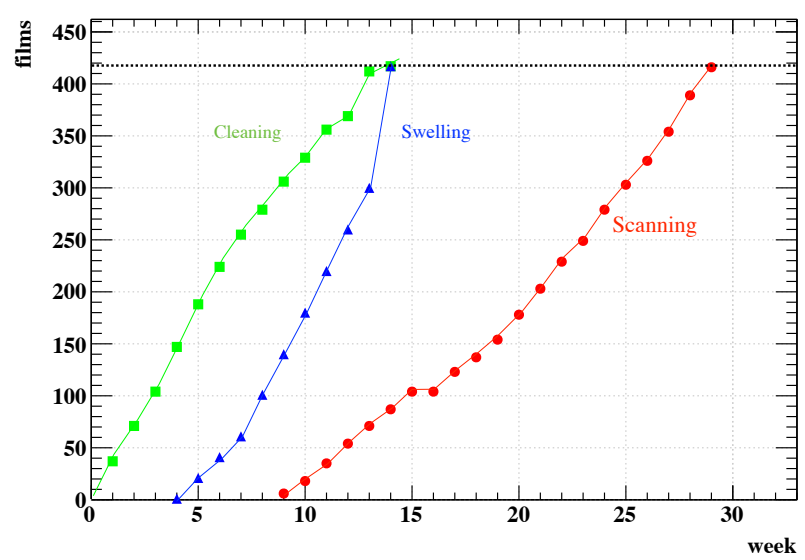

Figure 2: Progress in the data acquisition of converter films.

\subsection{Event reconstruction}

The reconstruction for gamma-ray events were processed using scan data. (The detailed description is appeared in reference [1]8]) Figure B] shows the result of event selection for a converter 
unit. In each converter film (area: $37.8 \mathrm{~cm} \times 25 \mathrm{~cm}$ ), $\sim 10^{8}$ tracks were readout in the angular range of $\left|\tan \theta_{X(Y)}\right|<1.3$, where $\tan \theta_{X(Y)}$ is the projection angle of the track, and the origin is the direction perpendicular to the film surface. To detect a start point (vertex) of gamma-ray event in a certain film, tracks that were reconstructed and not reconstructed in five down-stream films and three up-steam films, respectively, were selected, and the paired topology was required for each track. As a result, approximately $2 \times 10^{4}$ events were remained as gamma-ray event candidates in each converter film. Three dimensional views of reconstructed events that start at a film are displayed in figure $\Theta$. Momenta of electron and positron were reconstructed by measuring change of track angles at each film (the multiple Coulomb scattering method). The incident angle and energy of gamma ray were determined using momentum-weighted angles of electron and positron and sum value of the momenta, respectively.

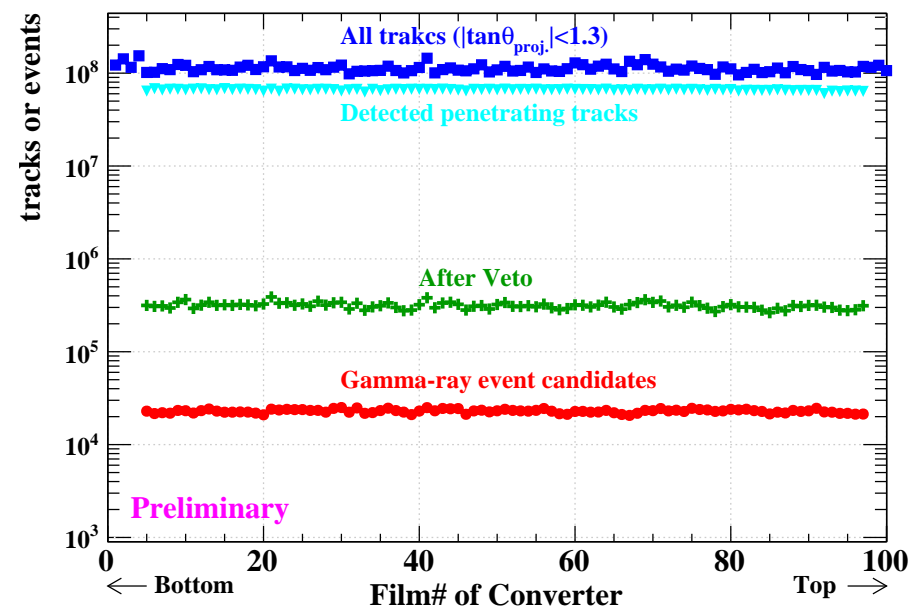

Figure 3: Result of gamma-ray event selection for a converter unit (\#4).

Red points on figure $\square$ show a preliminary result on the flux of vertical-direction events that were detected in the converter without time-stamp information. Currently, error bars conservatively account for $20 \%$ systematic uncertainty. Before the balloon flight, the emulsion telescope was on standby for 45 days on the ground, while the converter was directed to the zenith. Thus, the most of vertical events recorded in the converter are expected to be gamma rays on the ground. Blue line shows the predicted flux of atmospheric gamma rays on the ground, which was calculated by a Monte Carlo simulation.

\section{Gamma-ray Imaging Performance}

To evaluate the in-flight gamma-ray angular resolution of the emulsion telescope, we conducted the analysis using hadronic interaction events and their secondary gamma rays, which were occurred during observation at balloon altitudes [24]. As the left-hand schematic view in figure 6 , primary cosmic-ray hadrons interact with the converter itself and many secondary charged tracks are recorded in emulsion films. Such interaction events are clearly identified with their topologies, and the 3-D vertex positions can be determined with the resolution of several micron. In case of 


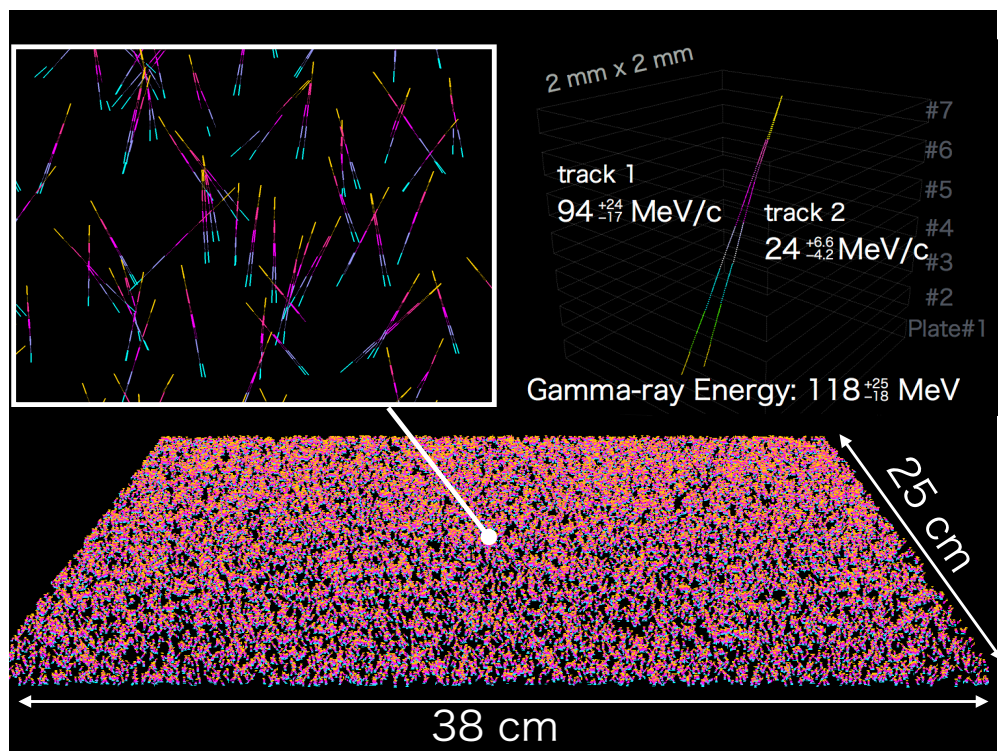

Figure 4: Three dimensional views of the gamma-ray events reconstructed at the converter. Tracks in different film numbers are indicated with different colors. The bottom pane shows the global view of events which starts from a film area. Top-left and top-left panes show close-up views.

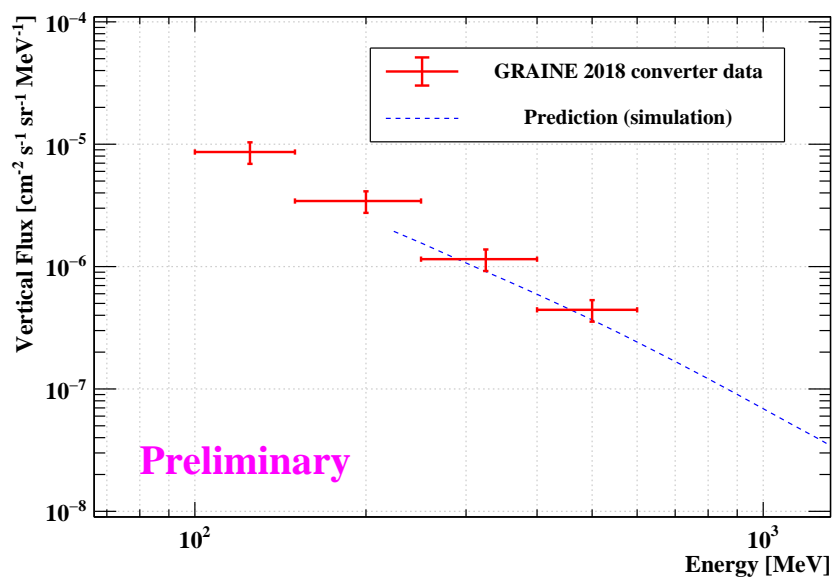

Figure 5: Red points indicate the flux of vertical-direction events that were detected in the converter without time-stamp information. Blue line shows the predicted flux of atmospheric gamma rays on the ground, which was calculated by a Monte Carlo simulation (using HKKM model).

the events that occurred at the up-stream of converter, $\sim 10 \%$ of their secondary gamma rays via $\pi^{0}$ decays are converted to electron-positron pair at the down-stream and detected. Because the lifetime of $\pi^{0}$ is much short, the gamma-ray direction precisely points at the interaction vertex point. Here, $\theta_{1}$ and $\theta_{2}$ were defined as the angle determined by the electron-positron pair tracks and the angle formed by connecting the point of the hadron interaction vertex to the point of the electron-positron vertex, respectively, and we evaluated the angular resolution with $\Delta \theta=\theta_{2}-\theta_{1}$.

The left-hand pane in figure 6 shows the 2-D distribution of $\Delta \tan \theta$ after the subtraction of chance coincidence background (the gamma-ray energy range: $100-300 \mathrm{MeV}$ ). The angular space 
was expressed with $\mathrm{R}$ and $\mathrm{L}$ projections, which are defined as the direction of gamma-ray slope and its orthogonal direction, respectively. A clear excess was observed at the region of $(0,0)$, and gamma-ray signals from hadronic interactions were detected. The angular resolution was evaluated by fitting the distributions of excess with gaussian on each projection. The resolutions at the angular regions of $0^{\circ}-27^{\circ}$ and $27^{\circ}-45^{\circ}$ were $0.57^{\circ}\left(\mathrm{R}: 0.36^{\circ}, \mathrm{L}: 0.44^{\circ}\right)$ and $0.81^{\circ}\left(\mathrm{R}: 0.72^{\circ}, \mathrm{L}: 0.37^{\circ}\right.$, respectively. We have confirmed that the emulsion gamma-ray telescope has excellent angular resolution during balloon flight and that the performance meets the requirements of the GRAINE2018 experiment (angular resolution requires less than $1^{\circ}$ in the $100-\mathrm{MeV}$ energy region).
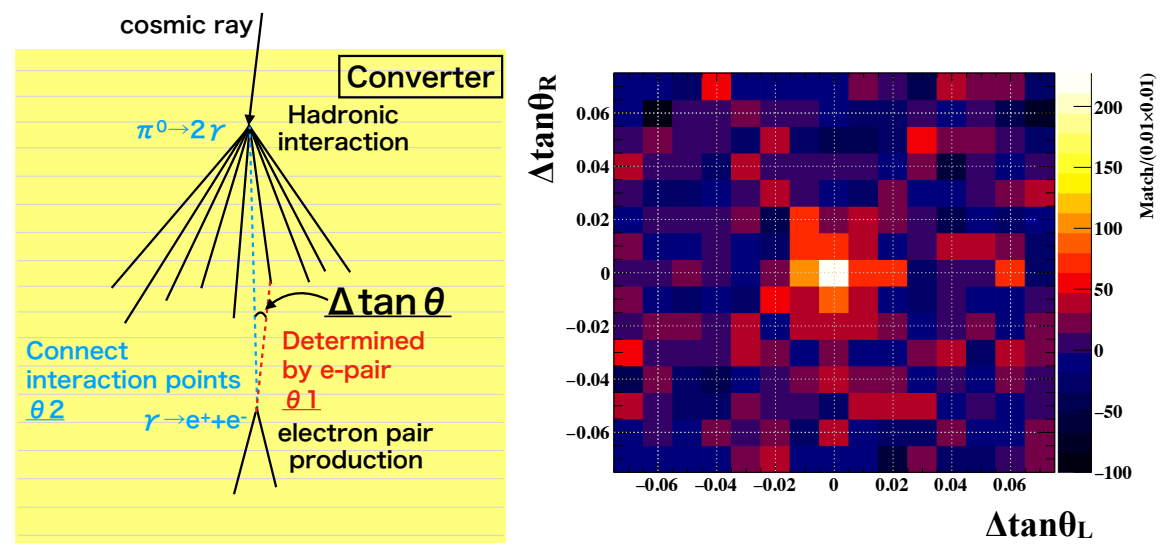

Figure 6: The right-hand schematic view shows a concept for evaluation of gamma-ray angular resoluton using hadronic interaction events and secondary gamma rays. The left-hand pane shows the 2-D distribution of $\Delta \tan \theta$ after the subtraction of chance coincidence background; 100-300 MeV gamma rays are used. [24]

\section{Summary and prospect}

We promote the cosmic gamma-ray observation project, GRAINE, with balloon-borne telescope utilizing the nuclear emulsion technique. The GRAINE 2018 experiment employed with 0.38- $\mathrm{m}^{2}$ emulsion telescope were performed on April 26, 2018, in Australia. The goals of this experiment are to detect the Vela pulsar, which is the brightest gamma-ray source in sub-GeV/GeV energy region, and to demonstrate the over-all performance of the balloon-borne emulsion telescope. The balloon flight including the period when Vela pulsar passed with in a field of view was succeeded, and then the recovery of payload and photofinishing process of flight films were completed without accident.

The data acquisition of converter films was performed with the emulsion readout system and the area of $\sim 40 \mathrm{~m}^{2}$ were scanned for 20 weeks. Currently, the process for gamma-ray event selection using $88 \%$ of the total data was completed. Through the analysis using hadronic interaction events and secondary gamma rays, it has been confirmed that the emulsion gamma-ray telescope has excellent angular resolution during balloon flight and that the performance meets the requirements of the GRAINE-2018 experiment (angular resolution requires less than $1^{\circ}$ in the $100-\mathrm{MeV}$ energy region). 
The analysis including time stamp and determination of attitudes is in progress and the report about Vela pulsar detection will be released soon. After the confirmation of over-all performance of the emulsion telescope, we will start the scientific observation with enlarged emulsion telescopes.

\section{Acknowledgments}

The scientific balloon (DAIKIKYU) flight opportunity was provided by ISAS/JAXA. We would like to thank Dr. M.Honda for calculating the atmospheric gamma-ray flux. This work was supported by JSPS, KAKENHI (grant numbers 17H06132, 18K13562, 18H01228).

\section{References}

[1] W. B. Atwood et al., Astrophys. J. 697, 1071 (2009).

[2] F. Acero et al., Astrophys. J. Suppl. Ser. 218, 23, (2015).

[3] T. Daylan et al., Phys. Dark Universe 12, 1 (2016).

[4] P. Gros et al., Astropart. Phys. 88, 30 (2017).

[5] A. Moiseev et al., PoS ICRC2017, 798 (2018).

[6] A. De Angelis et al., Exp. Astron. 44, 25 (2017).

[7] S. Takahashi et al., Adv. Space Res. 62, 2945 (2018).

[8] S. Takahashi, PhD thesis, Nagoya University, Japan (2011) (in Japanese).

[9] K. Ozaki et al., Nucl. Instrum. Meth. A 833, 165 (2016).

[10] H. Rokujo et al., PoS ICRC2015, 654 (2016).

[11] M. Morishita et al., PoS KMI2017, 073 (2017).

[12] Y. Nakamura st al., PoS KMI2017, 079 (2017).

[13] H. Rokujo et al., PoS KMI2013, 042 (2013).

[14] S. Takahashi et al., Prog. Theor. Exp. Phys. 2015, 043 H01 (2015).

[15] H. Rokujo et al., Nucl. Instrum. Meth. A 701, 127 (2013).

[16] S. Takahashi et al., Prog. Theor. Exp. Phys. 2016, 073F01 (2016).

[17] K. Ozaki et al., J. Instrum. 10, P12018 (2015).

[18] H. Rokujo, et al., Prog. Theor. Exp. Phys. 2018, 063 H01 (2018).

[19] S. Aoki et al., RADIOISOTOPES (In Japanese, Submitted).

[20] S. Takahashi et al., PoS ICRC2019, 607 (2019).

[21] H.Rokujo et al., J. Instrum. (Submitted).

[22] S. Takahashi et al., Nucl. Instrum. Meth. A 620, 192 (2010).

[23] M. Yoshimoto et al., Prog. Theor. Exp. Phys. 2017, $103 \mathrm{H} 01$ (2017).

[24] Y.Nakamura et al., PoS KMI2019, 039 (2019). 